\title{
Zu den Autoren
}

\section{B. Sc. Psych. Sandra Maria Ahlert}

arbeitet seit mehreren Jahren im Sonnenhof, Träger von inklusiver Jugend- und Eingliederungshilfe, und ist seit November 2018 dort als Koordinatorin mit einem Schwerpunkt in der Arbeit mit Menschen, die von FASD betroffen sind, tätig. Zudem ist sie zertifizierte Trainerin eines Gruppenangebotes für FASD-betroffene Jugendliche und Erwachsene.

E-Mail: ahlert@ev-sonnenhof.de

\section{Dipl.-Psych. Gela Becker}

ist psychologische Psychotherapeutin, langjährige Fachliche Leitung des Sonnenhofs. Aufbau des FASD-Fachzentrums, Entwicklung von Interventionen und Betreuungsangeboten, Mitarbeit an der S-3 Leitlinie zur Diagnostik der FASD, FASD-Fort- und Weiterbildung u.a. Trainerausbildung für FASDGesundheitskurse.

E-Mail: fasd-sprechstunde@ev-sonnenhof.de; www.fasd-fachzentrum.de

\section{Natalie Novick Brown, Ph.D.}

ist als Psychologin in den USA in den Staaten Washington und Florida zugelassen und hat sich in ihrer 20-jährigen Berufslaufbahn auf Fetale Alkoholspektrumstörungen, Entwicklungsstörungen und die Entwicklung von Kindern spezialisiert. Dr. Brown gründete und leitet FASDExperts (www. FASDExperts.com), eine multidisziplinäre Gruppe von Fachleuten, die forensische FASD-Evaluationen in den gesamten Vereinigten Staaten durchführt und ist klinische Assistenzprofessorin am Institut für Psychiatrie und Verhaltenswissenschaften der medizinischen Fakultät der Universität von Washington, USA.

E-Mail: drnataliebrown@gmail.com

\section{Dan Dubovsky, M. S. W. (Master of Social Work)}

hat sich als Diplom-Sozialpädagoge auf FASD spezialisiert und ist in einer Privatpraxis in Philadelphia, Pennsylvania, USA, tätig. Er ist Vater eines Sohnes mit FASD.

E-Mail: ddubovksy@verizon.net

\section{Andreas Francke}

Krankenpfleger/Dipl.-Heilpädagoge/Leitung sozialpsychiatrischer Wohnkonzepte (DGSP, Köln). Entwicklungs- und Führungsmanagement in Einrichtungen der Eingliederungshilfe für Erwachsene.

\section{Ulf Gerhardt}

ist Facharzt für Innere Medizin, Psychiatrie und Psychotherapie mit FASD Zertifikat der Universität Washington. Von 2011 bis 2014 war er Leitender Abteilungsarzt, von 2015 bis 2018 Chefarzt einer Suchtfachklinik. Außerdem verfügt er über einen ökonomischen Mastergrad der Universität Erlangen-Nürnberg und wurde 2017 zum Lehrbeauftragten der Hochschule Flensburg für strategisches Management ernannt. Seit dem 1.7.2018 ist er in eigener Praxis niedergelassen.

Email: Dr.gerhardt@hausarzt-luebbecke.com 


\section{Therese Grant, Ph.D.}

ist Erziehungswissenschaftlerin und Epidemiologin. Sie bekleidet die außerordentliche Ann Streissguth, Ph.D. Professur zu FASD am Institut für Psychiatrie und Verhaltenswissenschaften der medizinischen Fakultät der Universität von Washington, USA. Seit 2005 ist sie dort Leiterin der Abteilung FASD und Sucht und arbeitet in dieser Funktion international mit Wissenschaftlern und Klinikern in den Bereichen FASD-Screening, -Intervention, -Prävention und -Politikentwicklung zusammen. Als Expertin in Gesundheitsforschung und -intervention bei Risikogruppen mit verschiedenen kulturellen Hintergründen ist sie seit 2001 die Leiterin eines Eltern-Kind-Hilfsprogramms mit umfassenden Interventionsangeboten für Hochrisikomütter mit Subtanzmissbrauch, das inzwischen in mehreren Staaten der USA und in Kanada angeboten wird.

E-Mail: granttm@uw.edu

\section{Dorothea Hantelmann}

ist Ärztin für Neurologie und Psychiatrie. Sie war von 1981 bis $1989 \mathrm{im}$ vollstationären Bereich der Psychiatrie und Neurologie tätig, von 1989 bis zur Pensionierung im Sozialpsychiatrischen Dienst Berlin-Spandau. Dort hatte sie seit 1991 die Leitungsstelle inne. Seit 2014 ist sie im FASD-Fachzentrum des Sonnenhofs tätig.

\section{Prof. Dr. med. Klaus Hennicke}

ist Facharzt für Kinder-Jugendpsychiatrie und -psychotherapie, Dipl.-Soziologe und Familientherapeut und seit vielen Jahren mit der Thematik „Menschen mit Intelligenzminderung und psychischen Störungen“ befasst. Von 1995 bis 2014 war er stellvertretender Vorsitzender der „Deutschen Gesellschaft für seelische Gesundheit bei Menschen mit geistiger Behinderung“ (DGSGB). Von 2002 bis 2010 lehrte er Soziale Medizin an der Evangelischen Fachhochschule Bochum im Fachbereich Heilpädagogik. Über viele Jahre war er Leiter des Kinder- und Jugendpsychiatrischen Dienstes des Gesundheitsamtes Spandau von Berlin. Von 2005 bis 2016 hatte er den Vorsitz der Gemeinsamen Kommission „Inklusion und Intelligenzminderung“ der drei kinder- und jugendpsychiatrischen Fachverbände in Deutschland.

E-Mail: klaus.hennicke@posteo.de

\section{Prof. Dr. med. Annemarie Jost}

ist Fachärztin für Psychiatrie und Psychotherapie, systemisch lösungsorientierter Coach, marte meo Supervisorin. Sie war als Ärztin und Psychiaterin am St. Martinus Hospital Olpe und an der Rheinischen Landesklinik Bonn, tätig. Seit 1994 Professorin für Sozialpsychiatrie incl. Suchterkrankungen an der (Fach-)Hochschule Lausitz, seit 2013 an der BTU Cottbus-Senftenberg.

\section{Prof. Dr. rer. nat. Michael Klein}

ist Klinischer Psychologe und Psychotherapeut, langjährig in der Suchtforschung und Suchttherapie tätig mit Schwerpunkt „Sucht und Familie“, Leiter verschiedener Fachkliniken im Bereich Alkoholund Drogenabhängigkeit, seit 1994 Professor für Klinische Psychologie, Mental-Health- und Suchtforschung an der Katholischen Hochschule NRW, Köln. Leiter des Deutschen Instituts für Sucht- und Präventionsforschung (DISuP) und Past-Präsident der Deutschen Gesellschaft für Suchtpsychologie e. V. (dgsps).

E-Mail: Mikle@katho-nrw.de 
Priv. Doz. Dr. med. Dipl.-Psych. Mirjam N. Landgraf

ist Kinder- und Jugendärztin, Oberärztin, Psychologin, Leiterin der TESS-Ambulanz (für Risikokinder mit Toxinexposition in der Schwangerschaft) im iSPZ des Dr. von Haunerschen Kinderspitals der Universitätsklinik München, war Leitlinienkoordinatorin und -autorin der S3-Leitlinie zur Diagnostik der Fetalen Alkoholspektrumstörungen, ist Leiterin des Deutschen FASD KOMPETENZZENTRUMS Bayern und ist FASD-Beauftragte der Gesellschaft für Neuropädiatrie GNP.

E-Mail:mirjam.landgraf@med.uni-muenchen.de; www.ispz-hauner.de

\section{Dipl.-Soz. Päd. Gisela Michalowski}

ist diplomierte Sozialpädagogin, Vorsitzende der Selbsthilfegruppe FASD Deutschland e. V. und hat sich bereits jahrzehntelang sowohl im persönlichen als auch im professionellen Bereich für Menschen mit FASD engagiert.

E-Mail: info@fasd-deutschland.de

\section{Dipl.-Psych. Elke Rethmann}

ist Psychologin, Suchttherapeutin (VdR) sowie Supervisorin (DGSv) und Itd. Psychologin der Paracelsus-Wiehengebirgsklinik in Bad Essen, einer Rehabilitations-Fachklinik für die Behandlung von Abhängigkeitserkrankungen.

\section{Gila Schindler}

Studium der Sozialarbeit/Sozialpädagogik und Rechtswissenschaft. Fachanwältin für Sozialrecht in Heidelberg/Berlin, Schwerpunkt Eingliederungshilfe, Kinder- und Jugendhilfe sowie Pflege. Tätigkeit als Dozentin und Autorin.

\section{Dipl.-Psych. Ellen Scholz}

ist psychologische Psychotherapeutin mit Schwerpunkt FASD, Mitarbeiterin in der FASD-Beratungsstelle von 2007 bis 2010 im FASD-Fachzentrum des Sonnenhofs seit 2014, und führt Supervision und Fortbildung in Institutionen mit FASD-Betroffenen durch.

E-Mail: e.scholz@p-soft.de

\section{Sc. Psych. Lina Schwerg}

ist Psychologin und seit 2014 Mitarbeiterin des FASD-Fachzentrums des Sonnenhof e. V. mit einem Schwerpunkt in der Diagnostik von FASD bei Erwachsenen. Frau Schwerg ist Mitglied des Expert Advisory Committee für die jährlichen UBC FASD Research Konferenzen in Vancouver, Zertifizierte Trainerin eines Gruppenangebotes für Menschen, die von FASD betroffen sind und hält deutschlandweit Vorträge zum Thema FASD: Erkennung, Umgang und mögliche Interventionen. Frau Schwerg hat das erst deutschsprachige Screening für FASD für Erwachsen entwickelt und evaluiert, das Biographische Screeninginterview für FASD (BSI-FASD).

\section{Dipl.-Psych. Jessica Christine Wagner}

ist Diplom-Psychologin, Psychologische Psychotherapeutin/Verhaltenstherapie, Leiterin für therapeutischen Tanz (DGT) und arbeitete als wissenschaftliche Mitarbeiterin am Institut für Gesundheitspsychologie an der Europa-Universität Flensburg. Seit 10 Jahren ist sie national und international im Bereich FASD tätig und hat sich auf die neuropsychologische Diagnostik, Beratung und Therapie von Menschen mit FASD und vergleichbaren kognitiven Beeinträchtigungen spezialisiert.

E-Mail: je.wagner@keh-berlin.de 


\section{Dr. Theo Wessel}

ist Diplom Psychologe, approbierter Psychotherapeut (Verhaltenstherapie), Supervisor, Gesundheitswissenschaftler und Trainer Motivational Interviewing (MINT Inc.). Seine Arbeitsschwerpunkte: Suchtkrankenversorgung und Suchthilfeverbund, Psychoedukation bei Suchterkrankungen und Doppeldiagnosen, Geistige Behinderung und Sucht, Motivierende Gesprächsführung.

E-Mail: theowessel@t-online.de 